\title{
openheart Time to spontaneous ductus arteriosus closure in full-term neonates
}

\author{
Hiroyuki Nagasawa, ${ }^{1}$ Chikuma Hamada, ${ }^{2}$ Masashi Wakabayashi, ${ }^{2}$ Yuki Nakagawa, ${ }^{2}$ \\ Satoshi Nomura, ${ }^{2}$ Yoshinori Kohno ${ }^{1}$
}

To cite: Nagasawa $\mathrm{H}$, Hamada C, Wakabayashi M, et al. Time to spontaneous ductus arteriosus closure in full-term neonates. Open Heart 2016;3:e00413. doi:10.1136/openhrt-2016000413

Received 18 January 2016 Revised 17 March 2016 Accepted 12 April 2016

\section{CrossMark}

\footnotetext{
${ }^{1}$ Department of Neonatology, Gifu Prefectural General Medical Center, Gifu, Japan ${ }^{2}$ Department of Industrial Management \& Engineering, Tokyo University of Science, Tokyo, Japan
}

Correspondence to Dr Hiroyuki Nagasawa; hironag@ccn2.aitai.ne.jp

\section{ABSTRACT}

Objective: The mean closure time of the ductus arteriosus (DA) in full-term neonates is presumed to be 1-2 days after birth; however, whether this rate is accurate throughout the neonatal period is still unclear. In addition, the clinical determinants that influence DA closure remain unknown.

Methods: Echocardiography was performed 1826 times (897 in boys, 929 in girls) in 1442 participants (732 boys, 710 girls). An iE33 colour Doppler echocardiograph supplied by Philips Electronics was employed to examine DA flow. Data regarding sex, birth date, examination date, method of delivery, mother's age, past deliveries, neonatal body weight and body height were also collected. The Statistical Analysis System makes statistical clarification of these queries possible. We examined the persistence of DA in full-term neonates and appropriate for gestational age (AGA) neonates in the early neonatal period using colour Doppler echocardiography, and a subsequent analysis with SAS.

Results: After performing multivariable analyses, the median DA persistency times were 27.42 and $45.10 \mathrm{~h}$ after birth in boys and girls, respectively. A statistically significant sex difference was observed $(p<0.0001)$. Additionally, significant time differences were observed between vaginal and scheduled caesarean deliveries, at 26.97 and $28.93 \mathrm{~h}$, respectively $(\mathrm{p}=0.0245)$. No significant differences were observed in the other variables.

Conclusions: Spontaneous DA closure time curves were clarified for the first time throughout the early neonatal period in full-term and AGA neonates. It was revealed that both sex and delivery method play important roles in time to DA closure.

One of the most important fetal circulation components, the ductus arteriosus (DA) is destined to close immediately after birth. It is known that the DA in full-term neonates usually closes 1-2 days after birth. Gentile et $a l^{1}$ reported that the DA was undetectable in $42 \%$ of neonates by $24 \mathrm{~h}$ of age, and in $78 \%$ by $40 \mathrm{~h}$. The actual persistency rate of the DA through the neonatal period is not clear since it is quite difficult to detect the specific DA closure time in individual neonates. In order to detect the accurate closure

\section{KEY QUESTIONS}

What is already known about this subject?

- The mean closure time of the ductus arteriosus (DA) in full-term neonates is presumed to be $1-$ 2 days after birth; however, its accurate time is unknown.

What does this study add?

- Spontaneous DA closure time curves were clarified for the first time throughout the early neonatal period in full-term and appropriate for gestational age neonates.

- The median DA persistency times were 27.42 and $45.10 \mathrm{~h}$ after birth in boys and girls, respectively. A statistically significant sex difference was observed.

- Significant time differences were observed between vaginal and scheduled caesarean deliveries. The median DA persistency times were 26.97 and $28.93 \mathrm{~h}$ after birth, respectively.

How might this impact on clinical practice?

- Our results provide accurate data, which can be used in textbooks and when the DA closes in normal neonates.

time, echocardiography must be continuously performed in the neonate for 1 or 2 days, which is nearly impossible in clinical practice. Another method is to gather many participants and examine each at fixed, predetermined time points. Although certainly reliable and accurate, these data are not continuous. ${ }^{1}$

Survival analysis is used to analyse lifetime data, measured between a study's start and either when an event of interest occurs or if the event does not occur within the study period, at the latest examination. The latter data are called right-censored data and are usually analysed by the Kaplan-Meier method. ${ }^{2}$ The DA closure data, however, are a mixture of right-censored, left-censored and interval-censored data and seldom contain accurate closure times. The latter two cannot be analysed using the Kaplan-Meier method. The main reason we cannot have an accurate 
and continuous DA persistency rate is that there have been no statistical methods to deal with these three types of censored data. The Statistical Analysis System (SAS) LIFEREG (parametric maximum likelihood estimator for interval censoring data) and ICLIFETEST (nonparametric maximum likelihood estimator for interval censoring data) procedures allow statistical analysis of all censored data (right, left and interval) and can, therefore, analyse the DA data.

The SAS has programs which allow for detecting aspects influencing a targeted event. We chose some clinical factors that might influence the closure of DA. A multivariable analysis was performed to determine items that influence DA closure.

Colour Doppler images from conventional twodimensional (2D) echocardiography have been widely used to detect stenotic, regurgitating, and shunt flows in children. Colour Doppler has also been used to evaluate DA conditions in neonates. In this study, we examined DA in full-term and appropriate for gestational age neonate participants using colour Doppler echocardiography, and analysed the data according to the SAS procedure to obtain a DA closure time curve.

The objectives of our study were: (1) to acquire the persistency rate curve as per the hours after birth and (2) to identify significant clinical determinants that influence the closure of the DA.

\section{MATERIALS AND METHODS \\ Study design}

In order to determine the closure time curve of DA in normal neonates, a statistically regulated number of neonatal participants were enrolled in this study to ensure sufficient power. Each of the participants underwent 2D colour Doppler echocardiography at an irregular age (hours) after birth (table 1D). Some participants were followed until DA closure.

Data of DA closure for each participant only have the DA was persistent or not at the examination and the time the examination was performed. We divided the DA closure data into three types. In the first type, called the 'left', the DA has already closed at the first examination, and we usually draw the time course from the left to the right. The time of the event is located to the left of the time of the first examination. In the second type, called the 'interval-censored', the closure of DA is presumed to be between the two examinations. In the third one, called the 'right', the DA does not close during the examination period. It is presumed that the closure time of DA is after the examination period, and the closure time is to the right of the end of the examination period.

The SAS program is the only one program that can deal with these three kinds of censored data. Both a non-parametric procedure (ICLIFETEST) and an accelerated-failure time model assuming log-logistic

\begin{tabular}{|c|c|c|c|c|}
\hline \multicolumn{5}{|c|}{ (A) Clinical variables } \\
\hline & Boys & Girls & Total & p Valu \\
\hline $\begin{array}{l}\text { Number of } \\
\text { participants }\end{array}$ & $732(51)$ & $710(49)$ & 1442 & \\
\hline $\begin{array}{l}\text { Number of } \\
\text { examinations }\end{array}$ & $897(49)$ & $929(51)$ & 1826 & \\
\hline $\begin{array}{l}\text { Gestational } \\
\text { age (week) }\end{array}$ & $38.7(2.3)$ & $38.7(2.3)$ & & 0.34 \\
\hline Range & $37.0-41.9$ & $37.0-41.7$ & & \\
\hline $\begin{array}{l}\text { Birth weight } \\
\text { (g) }\end{array}$ & $2990(479)$ & $2890(448)$ & & \\
\hline Range & $2280-3870$ & 2225-3815 & & \\
\hline $\begin{array}{l}\text { Birth height } \\
\text { (cm) }\end{array}$ & $48.3(2.6)$ & $47.5(2.6)$ & & \\
\hline Range & $42.8-53.2$ & $43.1-52.7$ & & \\
\hline \multicolumn{5}{|c|}{ Parturient number } \\
\hline Primipara & $343(50)$ & $341(50)$ & 684 & \\
\hline Multipara & $389(51)$ & 369 (49) & 758 & 0.66 \\
\hline \multicolumn{5}{|l|}{ Delivery style } \\
\hline Vaginal & $424(50)$ & $419(50)$ & 843 & \\
\hline Caesarean & $308(51)$ & 291 (49) & 599 & 0.67 \\
\hline \multicolumn{5}{|c|}{ (B) Examination profile } \\
\hline \multicolumn{2}{|c|}{ Times of Examinations } & Boys & Girls & Tota \\
\hline \multicolumn{2}{|l|}{1} & 600 & 559 & $115 \varsigma$ \\
\hline \multicolumn{2}{|l|}{2} & 103 & 104 & 207 \\
\hline \multicolumn{2}{|l|}{3} & 25 & 33 & 58 \\
\hline \multicolumn{2}{|l|}{4} & 4 & 9 & 13 \\
\hline \multicolumn{2}{|l|}{5} & 0 & 3 & \\
\hline \multicolumn{2}{|l|}{6} & 0 & 2 & 2 \\
\hline
\end{tabular}

(C) Event occurrences and duration of observation

\begin{tabular}{lrrrr}
\hline & N & Minimum & Median & Maximum \\
\hline With event & 1204 & & & \\
$\quad$ Boys & $633^{*}$ & 7.67 & 62.60 & 167.62 \\
Girls & $571^{*}$ & 16.78 & 70.01 & 161.40 \\
Without event & 238 & & & \\
Boys & 99 & 2.12 & 28.85 & 148.57 \\
Girls & 139 & 4.30 & 38.88 & 137.95 \\
\hline
\end{tabular}

(D) Distribution of age at examination

\begin{tabular}{lc}
\hline Age (hour) & Number of examinations \\
\hline 0 to $<8$ & 41 \\
8 to $<16$ & 60 \\
16 to $<24$ & 128 \\
24 to $<32$ & 244 \\
32 to $<40$ & 133 \\
40 to $<48$ & 132 \\
48 to $<56$ & 232 \\
56 to $<64$ & 121 \\
64 to $<72$ & 118 \\
72 to $<80$ & 192 \\
80 to $<88$ & 91 \\
88 to $<96$ & 61 \\
96 to $<104$ & 103 \\
104 to $<112$ & 61 \\
112 to $<120$ & 26 \\
\hline
\end{tabular}


Table 1 Continued

(D) Distribution of age at examination

\begin{tabular}{lc}
\hline Age (hour) & Number of examinations \\
\hline 120 to $<144$ & 58 \\
$144<$ & 25
\end{tabular}

( ), IQR; ( ), percentage.

Medians are shown at gestational age, birth weight and birth height.

Unit, number of participant.

Unit, hours; ${ }^{*} p=0.0020$

distribution (LIFEREG), using SAS software, were used to analyse the data acquired by echocardiography.

\section{Study participants}

Study participants were all born at the Gifu Prefectural General Medical Center. We explained the aims of the study to the parents or guardians of the neonates and received their signed permission. The Ethics Committee of Gifu Prefectural General Medical Center approved this study in advance.

We considered neonates who met all of the following requirements to be 'normal': (1) gestational ages between 37 weeks 0 days and 41 weeks 6 days, (2) appropriate for gestational age, (3) no fetal distress and Apgar score more than 8 points at 5 min after birth, (4) no requirement for oxygen supplementation, respiratory or circulatory support, (5) no problems in sucking and urination, (6) no congenital heart disease, normal aortic and mitral valve function, and no evidence of coronary artery lesions.

The neonates who were born by emergency caesarean delivery, such as the cases which showed late deceleration on non-stress test were excluded; those who had apnoeas and/or bradycardia were also omitted.

Over a 51-month period, 1442 consecutive Japanese neonates (732 boys and 710 girls) were enrolled. The participant characteristics are shown in table 1A. The main indications of caesarean delivery were repeat caesarean delivery (44\%), pelvic and other abnormal fetal positions $(11 \%)$, placenta previa $(9 \%)$, twins $(7 \%)$, myoma uteri $(5 \%)$, cephalopelvic disproportion $(5 \%)$ and prolonged labour (4\%).

\section{Data acquisition}

The 2D utilised a 12-5 MHz iE33 transducer (Philips Electronics, Washington, USA), and was employed during natural sleep or calm conditions. Examination times were irregularly set. Persistent DA was defined as the detection of a parasternal colour flow image from the aorta to the pulmonary artery (PA) using Doppler echocardiography. The DA was examined in two directions from the pulmonary outflow tract (the main PA long-axis view, and the right and left pulmonary artery view). Some participants underwent multiple examinations until DA closure was detected. Once DA closure was detected, no further examinations were conducted.
A single observer $(\mathrm{HN})$ took all the measurements. The echocardiography examinations were performed 1826 times (897 in boys, 929 in girls) in 1442 participants. The distribution of the number of examinations for each participant is shown in table 1B. The observation durations are shown in table 1C. The distribution of age (hour) at examination is shown in table 1D. The event means to be detected are DA closure during the observation period. Although there were no significant differences between the sexes in the observation period, there was a significant difference in the number of events between sexes.

The participants' clinical information was collected from their outpatient or inpatient records. Body height (measured in the prone position), and body weight were measured at the time of echocardiography.

\section{Important premise}

The reader should recall that DA is present at birth, closes spontaneously and does not reopen afterwards.

\section{Statistical analyses}

The number of neonates needed for this study was determined according to Cohen's report. ${ }^{3}$ The effect size (the difference of means divided by $\mathrm{SD}$ ) was presumed to be 0.20. Statistical power was set at 0.85 and type 1 error in the two-sided test was allowed to be 0.05 . Accordingly, at least 450 examinations were needed in each group in order to accurately compare the mean values.

The F-test and Student $\mathrm{t}$ test, respectively, were employed to determine whether the sample variance and means of the two categories were statistically equivalent. The $\chi^{2}$ test was employed to determine whether the proportions of the samples were statistically equivalent. The LIFEREG procedure in the SAS (V.9.3; SAS Institute Inc, Cary, North Carolina, USA) software was employed to analyse the data on the accelerated-failure time model. The ICLIFETEST procedure was used to determine the presence of statistical differences, while Cox regression was used to compare clinical differences between the two categories. A multivariable analysis was performed in order to determine which clinical characteristics influenced DA closure. A p value of less than 0.05 was considered statistically significant.

\section{RESULTS}

The characteristics of the participants are shown in table 1. Between the sexes, there were no significant differences in group size, number of examinations, gestational age, parturient number or delivery methods. Additionally, there were no significant sex differences in the distribution of the number of examinations (table 1B). However, a significant sex difference $(p<0.0001)$ was identified in the proportion of DA closures.

The persistency rate curve had a sigmoid-like curve shape (figures 1-3). 
DA persistency rate

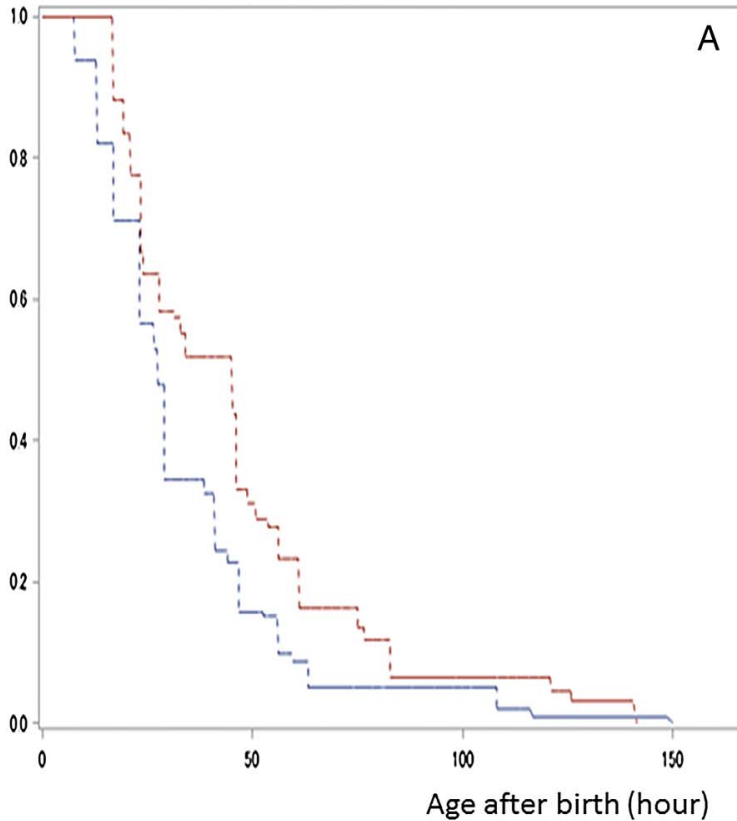

DA persistency rate

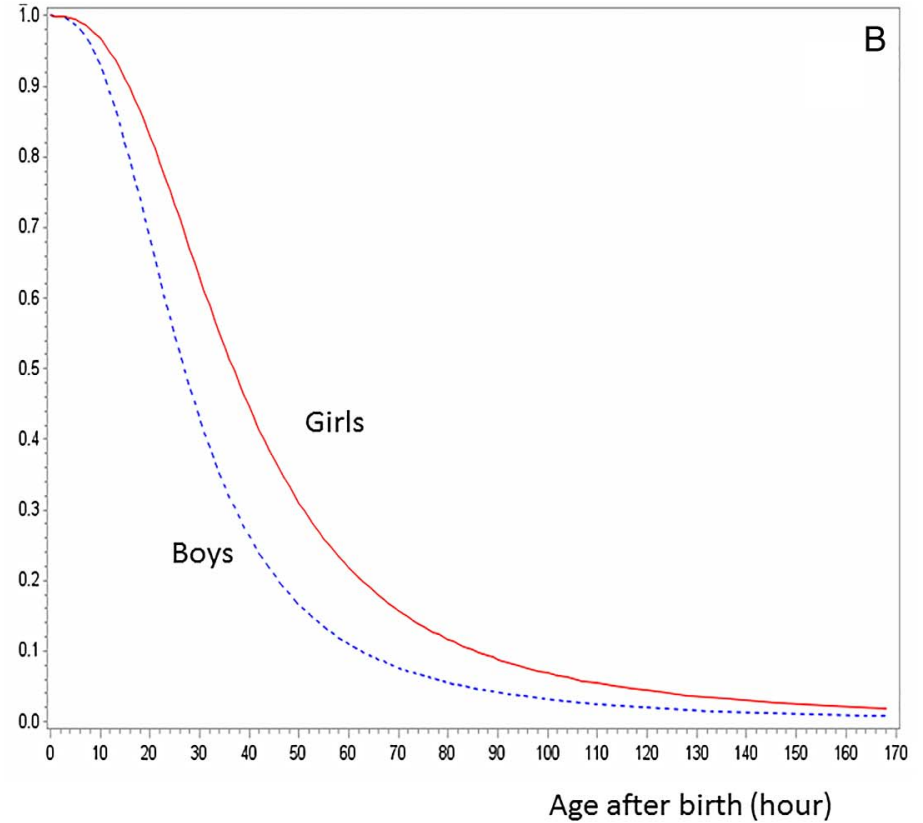

Figure 1 Ductus arteriosus closure time curve by sex $(p<0.0001)$. The life survival time curves were based on the hypothesised logarithmic logistic distribution (B using LIFEREG) and confirmed by the curves using ICLIFETEST (A). DA, ductus arteriosus.

The multivariable analyses of DA closure using the ICLIFETIME and LIFEREG procedures are shown in table 2. Significant differences between sexes $(p<0.0001)$ and delivery methods $(\mathrm{p}=0.0245)$ were noted, while there were no significant differences in the other clinical determinants.

\section{The sex difference}

The persistency rate curve in terms of sex was shown in figure 1 . Figure $1 \mathrm{~A}$ showed the non-parametric life survival time curve obtained with ICLIFETEST. The life survival time curves, hypothesising logarithmic logistic distribution and confirmed by the curves shown as
DA persistency rate

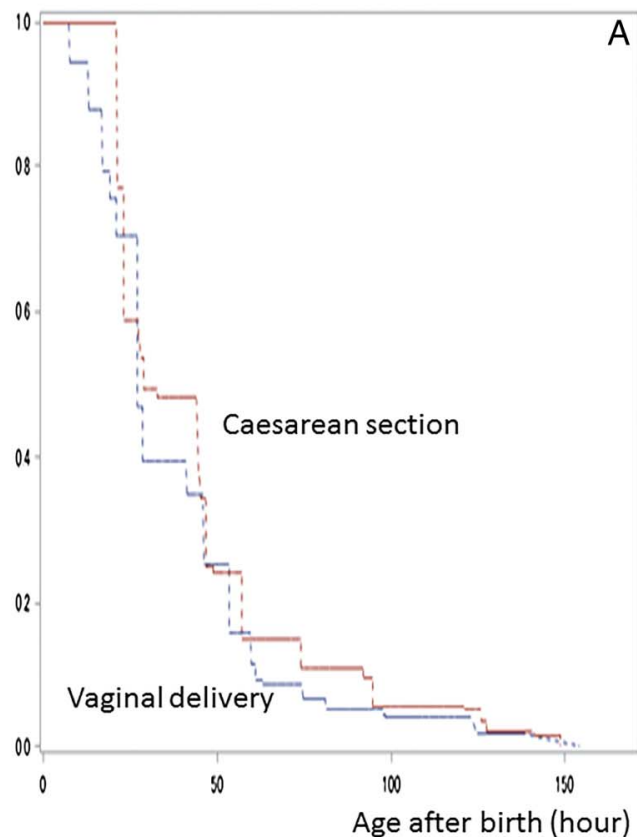

DA persistency rate

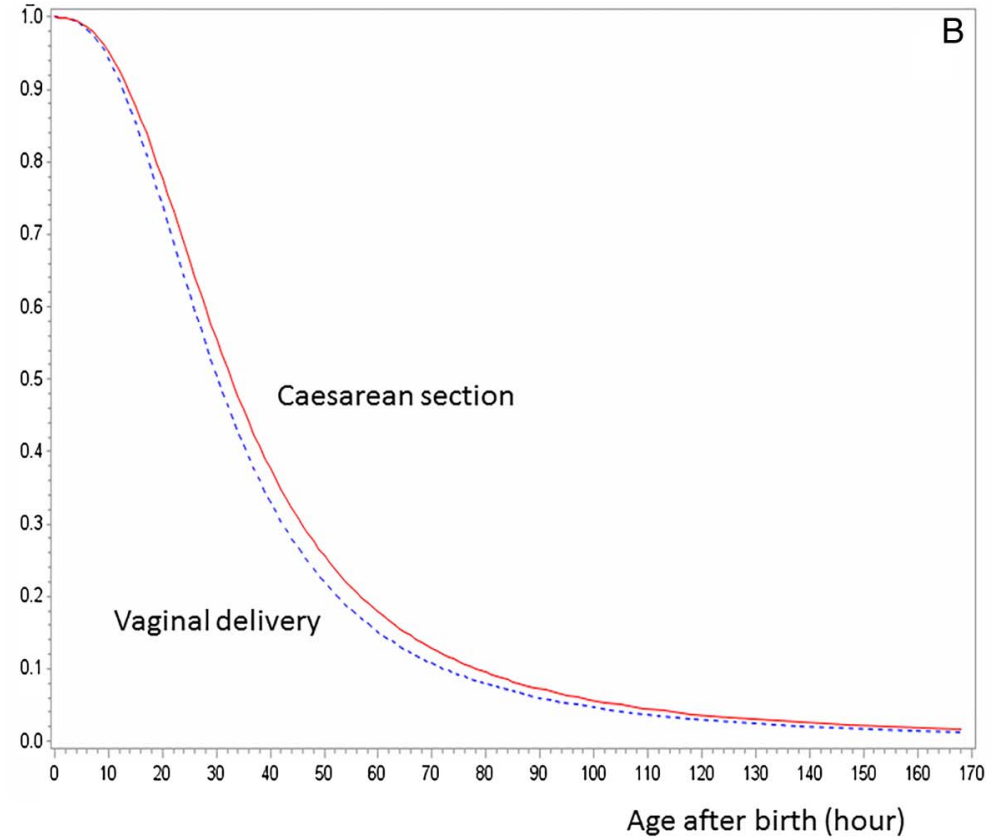

Figure 2 Ductus arteriosus closure time by delivery method $(p=0.0245)$. The life survival time curves, which were based on the hypothesised logarithmic logistic distribution (B using LIFEREG), and confirmed by the curves using ICLIFETEST (A). DA, ductus arteriosus. 
DA persistency rate

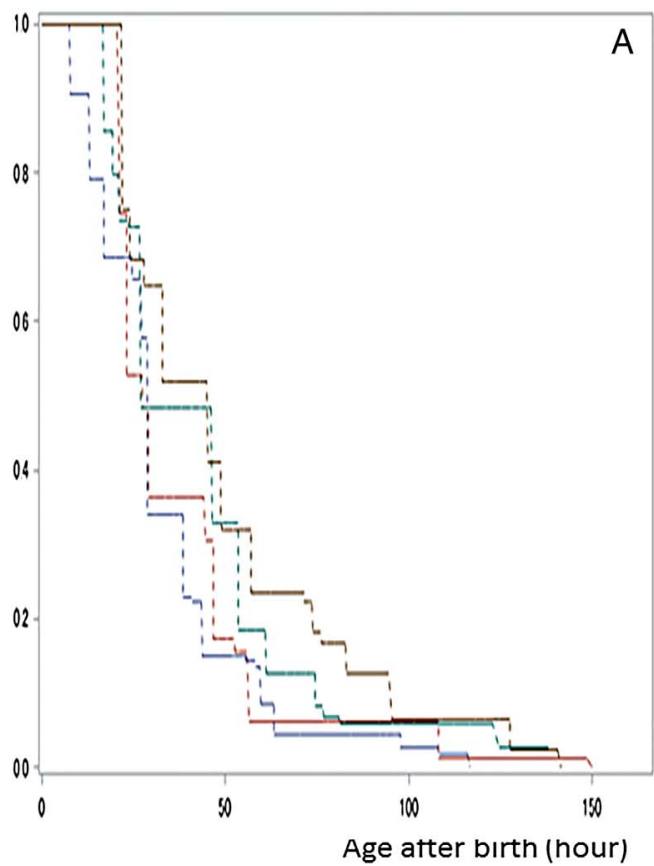

DA persistency rate

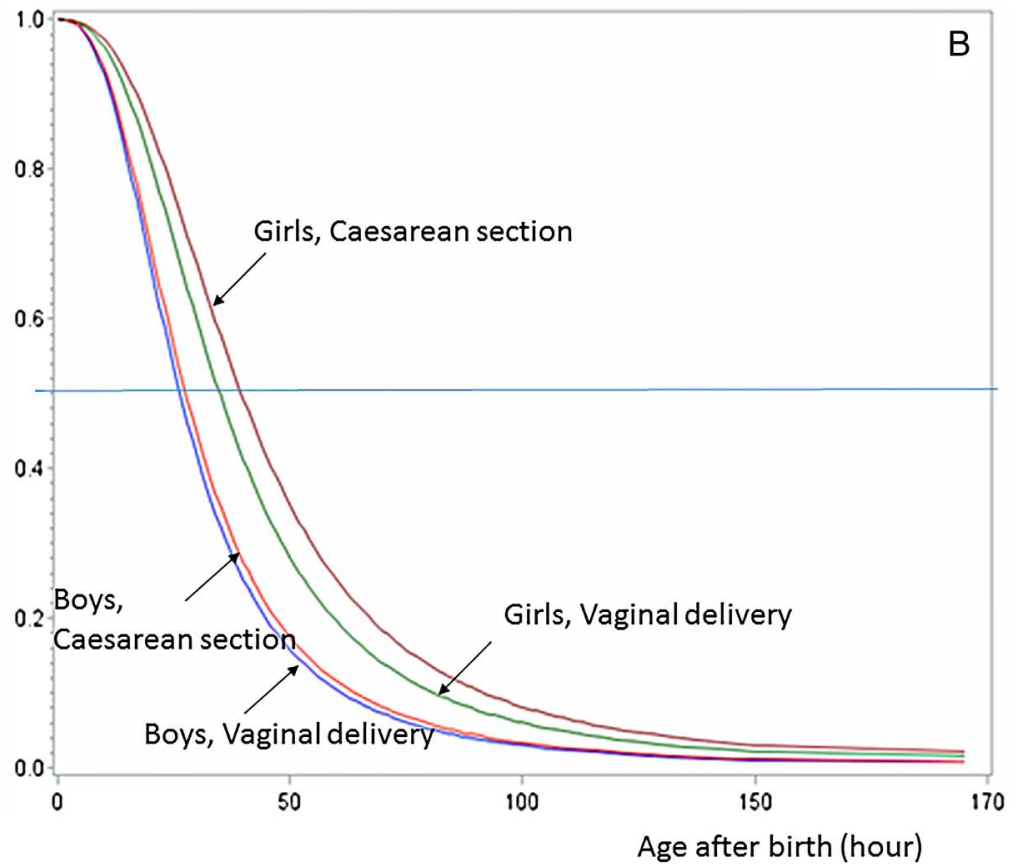

Figure 3 Persistency curves of boys and girls delivered vaginally and by caesarean delivery (summary). The life survival time curves were based on hypothesised logarithmic logistic distribution (B using LIFEREG) and confirmed by the curves using the ICLIFETEST (A). DA, ductus arteriosus.

figure 1A, were shown in figure 1B. The persistency rates in girls were higher than that of boys throughout the examination period $(\mathrm{p}<0.0001)$. The median DA survival times (MST) were 27.42 and $45.10 \mathrm{~h}$ after birth in boys and girls, respectively. The female:male ratio of MST was 1.645. The DA closure time in girls was significantly slower than that in boys.

\section{Comparison of DA closing time between vaginal and caesarean delivery}

The persistency rate curve in terms of delivery method is shown in figure 2. Figure 2A, B show the survival curves using ICLIFETEST and LIFEREG, respectively. Throughout the examination period, the persistency rates in neonates delivered via caesarean section were

Table 2 The multiple variance of coefficients in ductus arteriosus closing by ICLIFETEST (non-parametric) and LIFEREG (parametric)

\begin{tabular}{|c|c|c|c|c|}
\hline & & MST & & p Value \\
\hline & Number & ICLIFETEST & LIFEREG & (ICLIFETEST) \\
\hline Sex & & & & \\
\hline Female & 710 & 45.10 & 36.77 & \\
\hline Male & 732 & 27.42 & 26.89 & $<0.0001$ \\
\hline PN & & & & \\
\hline Primipara & 684 & 27.40 & 30.97 & \\
\hline Multipara & 758 & 30.30 & 31.61 & 0.6254 \\
\hline Method & & & & \\
\hline Vaginal & 843 & 26.97 & 30.25 & \\
\hline Caesarean & 599 & 28.93 & 32.75 & 0.0245 \\
\hline Height (cm) & & & & \\
\hline$\geq 48$ & 761 & 29.28 & 31.62 & \\
\hline$<48$ & 681 & 27.40 & 30.96 & 0.5612 \\
\hline Weight (g) & & & & \\
\hline$\geq 2500$ & 127 & 24.40 & 28.24 & \\
\hline$<2500$ & 1315 & 28.93 & 31.60 & 0.1620 \\
\hline GD (day) & & & & \\
\hline$\geq 272 \mathrm{~d}$ & 760 & 28.93 & 31.31 & \\
\hline$<273$ & 682 & 26.47 & 31.31 & 0.3626 \\
\hline
\end{tabular}

GD, gestational days; Height, body height; MST, median survival time; PN, parturient number; Weight, body weight. 
higher compared to the neonates who were delivered vaginally $(p=0.0245)$. The MST was 30.25 and $32.75 \mathrm{~h}$ after birth among the participants delivered vaginally and via caesarean section, respectively. The vaginal: caesarean delivery MST ratio was 0.932 . The DA closure time in the participants delivered vaginally was significantly faster than in those delivered via caesarean section.

The persistency curves of boys and girls delivered vaginally and abdominally are summarised in figure 3 . Figure 3A, B show the survival curves using ICLIFETEST and LIFEREG, respectively. The differences in DA closure between delivery methods were more clearly defined among the girls than the boys.

\section{DISCUSSION}

A precise mean time of the DA in full-term neonates has not yet been established. Moreover, no data are available regarding the accurate persistency rate throughout the neonatal period.

There are no programs which are able to deal with three kinds of censored data; 'left', 'interval' and 'right' censored, except the SAS program. These programs are based on the statistical method of the maximum likelihood estimation.

We applied multivariable regression analysis using the SAS programs to the data on DA closure time. We discovered successive persistency rate curves of DA throughout the early neonatal period and identified factors which influence the closure time for the first time.

The DA survival curves had sigmoid shapes. These results seemed reliable, since sigmoid curves frequently appear in many biological field situations, such as the increase in the population, as well as the relationship between death rate distribution and quantity of toxic agents, haemoglobin oxygen saturation and oxygen tension, and so on.

\section{Factors that influenced DA closure}

There were significant differences in sex and delivery method (table 2), and the $p$ value for sex $(p<0.0001)$ was lower than that for the delivery method $(\mathrm{p}=0.0245)$.

\section{Sex}

It has been reported that female patients have failures of DA closures about twice as often as male patients. ${ }^{45}$

Flinsenberg et al ${ }^{6}$ reported that oestrogen 17 $\beta$-estradiol induced an endothelium-independent relaxation of chicken DA. It is presumed that oestrogen may play an important role in the persistence of DA. No study has been reported on the theme in humans and therefore, the true reason is not known.

We reported a significant sex difference in the left ventricular end-diastolic volume index (body surface area calculated using the index formula reported by Haycock $e t a l^{7}$ ) in the early neonatal period using three- dimensional echocardiography. ${ }^{8}$ These results revealed that the sex differences seemed to already appear both morphologically and functionally.

\section{Delivery method}

Differences have been reported in the neonatal laboratory data and clinical conditions between the vaginal and caesarean delivery. However, there have been no reports on the relationship between the DA closure time and delivery method. This is the first report that examines these relationships.

Smith reported that prostaglandin E2 (PGE2) induces relaxation of the DA through the Gs protein adenyl cyclase. Increased cyclic adenosine monophosphate (cAMP) and PGE2 is one of main mechanisms of patency of DA. ${ }^{9}$ Interleukin-6 (IL-6) stimulates synthesis of PGE2. Mojaveri $e t a l^{10}$ reported that mean serum IL-6 levels in neonates delivered vaginally $(10.9 \mathrm{pg} / \mathrm{mL})$ were higher than those in neonates delivered by elective caesarean section $(6.6 \mathrm{pg} / \mathrm{mL})$, but the difference was not significant $(p=0.86)$. The relationship between serum IL-6 level and DA closure is still unclear.

Using full-spectrum near-infrared spectroscopy, Isobe et $a l^{11}$ reported that the cerebral oxygenation in neonates delivered abdominally significantly decreased compared to that in neonates delivered vaginally at $8.5 \mathrm{~min}$ post-delivery. Urlesberger $e t a l^{12}$ reported that arterial oxygen saturation and heart rate were significantly lower in infants delivered abdominally than in those delivered vaginally. Noh et $_{a l} \mathrm{l}^{13}$ examined oxidative markers in the umbilical cord blood and reported that the markers in neonates who were delivered through planned caesarean section were significantly higher compared to those in infants delivered vaginally. These reports indicate that neonates delivered abdominally are at greater risk for problems related to their respiratory or circulatory systems.

\section{Other covariates}

No significant relationships were seen between DA closure and the parturient number, body weight, body height or gestational days (table 2).

\section{Study limitations}

The DA closure time curves are based on all participants' data and not on individual participant data. It is impossible to expect an exact DA closure time for each neonate.

\section{Application of the SAS programs to other fields}

The life data analysis used in this paper could potentially be applied to many diseases. Lately, interest has grown in progression-free survival for slowly progressive diseases that are difficult to cure (Y So, G Johnston, SH Kim. Analyzing interval-censored survival data with SASTM software. SAS global forum 2010). ${ }^{14}{ }^{15}$ Progression-free survival is often used as a clinical end point in randomised controlled trials for some slowly progressive 
cancer therapies; however, there has been no way to study the morbidity rate and age of onset in patients with these diseases. The procedure used in this paper could be usefully applied to the analysis of these diseases.

Another advantage to the SAS program is the ability to freely choose examination intervals. Examining many patients at particular fixed intervals or ages can be a burden to examiners. It is preferable that examination intervals be conducted irregularly and still produce an accurate survival curve.

\section{CONCLUSION}

Using DA closure data acquired by echocardiography and analysed through SAS programs, successive persistency rate curves of DA throughout the early neonatal period were revealed. Factors that influence the closure time were identified for the first time. These results could lead to new progressions in the endocrinological, neurological or other fields of the early neonatal period.

Acknowledgements The authors would like to give special thanks to our colleagues Yutaka Yamamoto, Masashi Kondo, Masami Sugawara, Toshinari Koyama, Daisuke Terazawa and Ryosuke Miura for their indirect support in examining the participants on echocardiography and analysing their DA data.

Collaborators HN examined all the participants and drafted the work. YK supervised the writing of the paper and gave the final approval. $\mathrm{CH}, \mathrm{MW}, \mathrm{YN}$ and SN analysed the data using SAS programs.

Competing interests None declared.

Patient consent Obtained.

Ethics approval The Ethics Committee of Gifu Prefectural General Medical Center.

Provenance and peer review Not commissioned; externally peer reviewed. Data sharing statement No additional data are available.

Open Access This is an Open Access article distributed in accordance with the Creative Commons Attribution Non Commercial (CC BY-NC 4.0) license, which permits others to distribute, remix, adapt, build upon this work noncommercially, and license their derivative works on different terms, provided the original work is properly cited and the use is non-commercial. See: http:// creativecommons.org/licenses/by-nc/4.0/

\section{REFERENCES}

1. Gentile R, Stevenson G, Dooley T, et al. Pulsed Doppler echocardiographic determination of time of ductal closure in normal newborn infants. J Pediatr 1981;98:443-8.

2. Kaplan EL, Meier P. Non-parametric estimation from incomplete observations. J Am Statist Assoc 1958;53:457-81.

3. Cohen J. Statistical power analysis for the behavioral sciences. 2nd edn. Hillsdale, NJ: Lawrence Erlbaum Associates, 1988:19-74.

4. Sampayo F, Pinto FF. The sex distribution of congenital cardiopathies. Acta Med Port 1994;7:413-18.

5. Samánek M. Boy:girl ratio in children born with different forms of cardiac malformation: a population-based study. Pediatr Cardiol 1994;15:53-7.

6. Flinsenberg TW, van der Sterren S, van Cleef AN, et al. Effects of sex and estrogen on chicken dustus arteriosus reactivity. $A m \mathrm{~J}$ Physiol Regul Integr Comp Physiol 2010;298:1217-24.

7. Haycock GB, Schwartz GJ, Wisotsky DH. Geometric method for measuring body surface area: a height-weight formula validated in infants, children, and adults. J Pediatr 1978;93:62-6.

8. Nagasawa $\mathrm{H}$. Evaluation of left ventricular volumes in the early neonatal period using three-dimensional echocardiography. Cardiol Young 2014;24:685-93.

9. Smith GC. The pharmacology of the ductus arteriosus. Pharmacol Rev 2006;50:35-58.

10. Mojaveri MH, Mohammadzadeh I, Bouzari ZAS, et al. The comparison of serum interleukin- 6 of mothers in vaginal and elective cesarean delivery. Caspian J Intern Med 2014:5:223-6.

11. Isobe K, Kusaka T, Fujikawa $Y$, et al. Measurement of cerebral oxygenation in neonates after vaginal delivery and cesarean section using full-spectrum near infrared spectroscopy. Comp Biochem Physiol A Mol Integr Physiol 2002;132:133-8.

12. Urlesberger B, Kratky E, Rehak T, et al. Regional oxygen saturation of the brain during birth transition of term infants: comparison between elective cesarean and vaginal deliveries. $J$ Pediatr 2011:159:404-8.

13. Noh EJ, Kim YH, Cho MK, et al. Comparison of oxidative stress markers in umbilical cord blood after vaginal and cesarean delivery. Obstet Gynecol Sci 2014;57:109-14.

14. Gentleman R, Geyer CJ. Maximum likelihood for interval censored data: consistency and computation. Biometrika 1994;81:618-23.

15. Kalbfleisch JD, Prentice RL. The statistical analysis of failure time data. New York: John Wiley \& Sons, 1980:52-94 and 218-246. 